\title{
THE EFFECT OF NEUROTROPIN UPON THE HYPERTHYROIDISM AND BASEDOW'S DISEASES \\ by
}

Yoshitada TAKINO

(Dainippon-Zoki Kenkyusho, Osaka, Japan. Director: Masuichi Takino)

By chance Dr. Yoshiro Kondo has found that the symptom of hyperthyroidjsm combined with asthmatic bronchitis has become better by the treatment of Asthremedin and has kindly reported this fact to me. He asked me why Asthremedin was effective upon not only asthmaattack, but also upon hyperthyroidism. Then 1 answered that perhaps Neurorropin acted upon it, because it is contained in Asthremedin and it can calm the stimulated condition of all kinds of nerve certainly. After that time 1 had the chance to treat the typical Basedow's diseases with Neurotropin and certified Dr. Kondo's report.

The result was the following:

Case 1. This case was reported by Dr. Kondo. 16 year old girl- Diagnosis: Hyperthyroidism and asthmatic bronchitis, especially during the menstruation. Heredity : father is suffering from hypertension and bronchial asthma. One brother has tuberculosis. Past diseases of this girl: often urticaria. Present state: she has found unexpectedly the swelling of the neck since 10 years. Until two yaars ago she had no pain, but now she is very tired and became somewhat lean. Furthermore she complained about the want of appetite and temperature 37.2. Pulse 110 in a minute. At the time of the first examination she had heart acceleration and shining eye, but another eye symptom and tremor of four extremities is not seen. The swelling of her thyroid gland is easily seen. It's hardness is medium. The course of this disease: she was treated at first by methiosil during 3 months, but in vain. Dr. Kondo began to use Asthremedin for the cure of asthmatic bronchitis 6 months after the first treatment. 12 ampouls of asthremedin were used, and she had no more asthmatic bronchitis. Besides that it was wonderful that it was unable to be seen by the naked eye. Of course the swelling could barely be touched even when her nape was bended backwards. At the same time heart accele- 
ration and shining eye disappeared completely.

Case 2. 46 year old man. Heredity: father is suffering from bronchial asthma. Past disease. nothing. Present state: since half year ago he had the tremor of four extremities, especially of hands, heart acceleration and tachycardia. Since before a month he found the swelling of the neck. At the time of first examination he showed the wet skin, tachycardia (120130 in a minute), blood pressure 135-60 and tremor of 4 extremities and higher half body, the decrease of strength of four extremities and his eyeballs projected. The swelling of his thyroid gland had spread to $M$. sternocleidmastoideus. Its hardness medium. Base-metabolism was not measured. An artificial asthma-attack (deep respiration and choking feeling) occured by Czermak's test (carotid sinus press test). Pulse became slow by Ashner's test. The course fo this disease : after $15 \mathrm{amp}$. of Neurotropin Special were used every other day. Then neither tremer nor heart acceleration appeared any more. And walking became very easy and the strength of his under-extremities was recovered. The swelling of the thyroid gland reduced eminently. Pulse became 90 . But only skin remained as it was. Now he can work.

The mechanism of action of Neurotropin upon this disease is not yet known. But it is already well known, that in this disease the instability of the autonomic nervous system, especially the stimulated condition of sympathicus, and in some case even the stimulated condition of parasympathicus appear. Upon this condition Neurotropin is able to exert an effective action. 